\title{
Successful maternal and fetal outcome of Guillain-Barre syndrome complicating pregnancy: a case report
}

\author{
Saunitra A. Inamdar ${ }^{1}$, Anil H. Inamdar ${ }^{2}$, Richa Chaudhary ${ }^{1}$, Vaidehi S. Subhedar ${ }^{1}$
}

${ }^{1}$ Department of Obstetrics and Gynaecology, ${ }^{2}$ Department of Medicine, Acharya Vinoba Bhave Rural Hospital, Sawangi (Meghe), Wardha, Maharashtra, India

Received: 13 July 2013

Accepted: 4 August 2013

*Correspondence:

Dr. Richa Chaudhary,

E-mail: richachaudhary17@gmail.com

(C) 2013 Inamdar SA et al. This is an open-access article distributed under the terms of the Creative Commons Attribution Non-Commercial License, which permits unrestricted non-commercial use, distribution, and reproduction in any medium, provided the original work is properly cited.

\section{ABSTRACT}

Guillain-Barré syndrome rarely complicates pregnancy, incidence being 2/100000 population. We report a case of 23year-old 2nd gravida who developed a progressive ascending paralysis during the second trimester of pregnancy. The worsening of the respiratory insufficiency required transfer to an intensive care unit but did not require artificial ventilation. Cerebrospinal fluid examination revealed albumin-cytological dissociation and repeated electrophysiological studies showed typical features of demyelinating neuropathy with conduction blocks. Biological investigations, especially CMV and campylobacter jejuni serologies, were all negative. Intravenous immunoglobulin infusions resulted in rapid improvement in the patient. This case raises the question of the relationship between the Guillain-Barré syndrome and pregnancy. The occurrence of the disease, as well as the rapid recovery in antenatal period could be consecutive to a partial failure of the maternal immunological tolerance toward the fetus.

Keywords: Guillain-Barré syndrome, Plasmapheresis, Immunoglobulins

\section{INTRODUCTION}

Guillain-Barré syndrome (GBS) is an acute polyneuropathy, a demyelinating disorder affecting the peripheral nervous system. Ascending paralysis, weakness beginning in the feet and hands and migrating towards the trunk, is the most typical symptom. It can cause life-threatening complications, in particular if the respiratory muscles are affected. The disease is usually triggered by an infection. The Guillain-Barre syndrome rarely complicates pregnancy. The incidence in pregnancy is $2 / 100000$ population. $^{2}$

Pregnancy and delivery are unaffected by GB syndrome. ${ }^{3}$ There is no increase in spontaneous abortion. Increased incidence of respiratory complications is mostly due to the gravid uterus. Mechanical ventilation due to GB syndrome: $33 \%$ in pregnant vs. $16 \%$ of non pregnant patients. Mortality due to GB syndrome is doubled if contracted in third trimester. Remote history of GB is associated with 5\% risk of relapse. Despite neurological deficits, impairment of uterine contraction activity is not there and vaginal delivery can be well established. Therefore, maternal GBS is not an indication for cesarean section and operative delivery should be reserved for obstetric indications only.

Herein, we report a patient of GB syndrome who presented in the second trimester of pregnancy.

\section{CASE REPORT}

A 23 -year-old $2^{\text {nd }}$ gravida was referred to the obstetric unit of AVBR Hospital at 33 weeks gestation complaining of difficulty in breathing since 2 hours and inability to walk since 1 day, B/l limb weakness had started 2 weeks ago and was becoming progressively worse. There was history of a recent viral fever. She didn't complain of urinary incontinence and difficulty in swallowing.

Physical examination revealed a well-nourished young woman who was fully conscious and alert. All vital signs were normal; with no evidence of respiratory distress. 
No cranial nerves were involved. Motor examination revealed quadriparesis, muscle weakness being more marked in the proximal muscles than in the distal group. The deep tendon reflexes were absent. Sensations were normal Obstetric examination revealed that the height of the uterine fundus corresponded to the stage of gestation, with a single foetus presenting cephalically. The fetal heart beat was present. Vaginal examination revealed a normal gynaecoid pelvis; and the internal os was closed.

Ultrasonography was done suggesting the liquor volume was normal and the estimated weight of the foetus was $2250 \mathrm{~g}$. NST was done which was reactive.

Albuminocytologic dissociation in cerebrospinal fluid with elevated protein content and the characteristic electrophysiological abnormalities on nerve conduction studies confirmed the diagnosis of GB syndrome. Biological investigations, especially CMV and Campylobacter jejuni serologies, were all negative.

Therapy with intravenous immunoglobulin infusion was initiated. The patient was managed in the respiratory intensive care unit. She did not require mechanical ventilation.

There was gradual improvement in her muscle power and the patient had full recovery. At term, she progressed spontaneously into labour and delivered vaginally. The labour did not require augmentation with oxytocin and a healthy $2500 \mathrm{~g}$ male baby was delivered.

Obstetrically; the patient had an uncomplicated puerperium and did not require mechanical ventilation. Patient received physiotherapy and doesn't have any disability.

\section{DISCUSSION}

It is of opinion that the management of the gravid patient with Guillain-Barre syndrome does not differ much from that in non-pregnant patients with this disease. Supportive care in an intensive care unit remains the cornerstone of treatment. Management of airway and respiratory infection, maintaining fluid and electrolyte balance, nutritional support and effective rehabilitation are essential.

Unnecessary obstetric intervention must be strongly resisted. In this case, the patient did not require mechanical ventilation at the time of delivery. It was reported that as many as $34.5 \%$ of women suffering from GBS during pregnancy required ventilatory support and the maternal mortality exceeded $10 \%{ }^{1}$

The progress of labour in the case described was no different and there was no evidence to suggest that uterine function was altered. It was our plan to have a stricter criteria before resorting to caesarean section. Nelson and McLean ${ }^{1}$, in a review of the syndrome, state that the condition is not affected by pregnancy or pregnancy termination.

Recently, plasmapheresis has been found to be of value in Guillain-Barre syndrome if performed within 7 days of the onset of symptoms and signs and in patients with severe disease.

Plasmapheresis has been used in pregnant patients with rhesus haemolytic disease and in severe cases in pregnancy.

It was not attempted in our patient because she was first seen 20 days after the onset of symptoms and she had a milder course of disease. A multidisciplinary approach involving the physicians and obstetricians is essential in the management of GBS in pregnant women.

Attention should also be paid to the identification and treatment of infective complications, prevention of venous thromboembolism, pain management, and the management of psychological distress resulting from the disease and anxiety towards the pregnancy. Apart from pharmacological prophylaxis, physical measures such as pressure stockings, physiotherapy and early mobilization are also useful.

Nosocomial infection is an important complication of GBS patients. As many as $25 \%$ of GBS patients acquired pneumonia and $30 \%$ suffered from urinary tract infections. ${ }^{3}$ Because these infections tend to be more severe in pregnant women, early identification and treatment are desirable.

To conclude, early diagnosis and prompt intensive multidisciplinary supportive care followed by IVIG therapy is advantageous in a GBS-complicated pregnancy and improves the prognosis for both mother and fetus.

\section{REFERENCES}

1. Nelson LH, McLean WT. Management of LandryGuillain-Barre' syndrome in pregnancy. Obstet Gynecol 1985;65:25S-29S.

2. Ravn H. The Landry-Guillain-Barre syndrome. A survey and a clinical report of 127 cases. Acta Neurol Scand 1967;43:Suppl 30:1-64.

3. Ropper AH. The Guillain-Barre syndrome. N Engl J Med 1992;326:1130-6.

DOI: $10.5455 / 2320-1770$. ijrcog20130952

Cite this article as: Inamdar SA, Inamdar $\mathrm{AH}$, Chaudhary R, Subhedar VS. Successful maternal and fetal outcome of Guillain-Barre syndrome complicating pregnancy: a case report. Int J Reprod Contracept Obstet Gynecol 2013;2:478-9. 